Research Article

\title{
Graduating from Medical School amid a Pandemic: A Study of Graduates' Mental Health and Concerns
}

\author{
Hend M. Alkwai $\mathbb{D}$ \\ Department of Pediatrics, College of Medicine, University of Ha'il, Ha'il, Saudi Arabia \\ Correspondence should be addressed to Hend M. Alkwai; h.alkwai@uoh.edu.sa
}

Received 9 September 2020; Revised 22 November 2020; Accepted 15 January 2021; Published 28 January 2021

Academic Editor: Ahmed Rachid

Copyright ( $\odot 2021$ Hend M. Alkwai. This is an open access article distributed under the Creative Commons Attribution License, which permits unrestricted use, distribution, and reproduction in any medium, provided the original work is properly cited.

\begin{abstract}
The corona virus disease of 2019 (COVID-19) pandemic has vastly impacted individuals worldwide. Millions have contracted the disease thus far; however, the ramifications of containment measures such as quarantine and lockdown have affected many more. This study aimed to explore the prevalence of self-reported anxiety and depression among a cohort of Saudi medical students, as well as graduating medical students' concerns, during this pandemic. This cross-sectional study utilized an online survey that collected demographic data, scores on validated anxiety, and depression scales, as well as the graduates' concerns. The Generalized Anxiety Disorder 7-item (GAD-7) and the Public Health Questionnaire 9-item (PHQ-9) scales were used. Cutoff scores for anxiety symptoms were (GAD-7 $\geq 8$ ) and for depression (PHQ-9 $\geq 10$ ). Out of 83 graduates, 55 completed the online survey. Anxiety symptoms were present in $34 \%$, depression was present in $26 \%$, and $19 \%$ had both depression and anxiety symptoms. Female graduates experienced higher anxiety symptoms ( 54 vs. $15 \%, p=0.004$ ). The highest-ranked concerns were the possible effect of the pandemic on the forthcoming internship year and on the impact on the current academic course. We found high levels of anxiety and depression symptoms during the pandemic among our cohort of medical students in their final year of study. Our findings suggest that medical students in their final years may be negatively affected by the pandemic, where high levels of depression and anxiety coincide with concerns about the effect of the pandemic on the next phase of their vocation.
\end{abstract}

\section{Introduction}

Corona Virus Disease 2019 (COVID-19) was the term coined by the World Health Organization (WHO) to the disease that was first reported as a cluster of pneumonia cases in Wuhan City, Hubei province, China in December 2019 [1]. The disease was declared a global pandemic by the WHO on March 11, 2020 [2]. Concerns relating to mental health during the pandemic quickly surfaced, supported by several studies addressing the mental health of the quarantined public, infected patients and their relatives, workers (particularly healthcare workers), and students.

In their efforts to combat the COVID-19 pandemic, many countries have implemented lockdown procedures that have affected learners worldwide. According to the United Nations Educational, Scientific, and Cultural Organization (UNESCO), more than 1.3 billion students in 136 countries were affected by mid-April 2020 [3]. Medical education was not immune to this affection [4]. Views have varied on how medical schools should respond to the resultant closures and training restrictions, with the majority advocating the involvement of medical students in the fight against the pandemic [5]. Student-led initiatives to help combat the pandemic were seen as well $[6,7]$.

The academic and emotional demands of medical education on students are well-known. A recent meta-analysis that included studies comprising more than 40000 medical students has shown that the global prevalence rate of anxiety was $33.8 \%$, with increased rates reported from Asia and the Middle East [8]. Another meta-analysis that pooled results from 167 cross-sectional studies showed that the prevalence of depression or depressive symptoms was $27.2 \%$ [9].

As the mental toll of the pandemic seems varied and widespread, this study aimed to explore the mental health of a susceptible population. Medical students in their last year of studies who were less than two months away from 
graduation when closures took effect were surveyed for the presence of anxiety and depressive symptoms.

\section{Materials and Methods}

Graduating medical students in their last year of study were recruited. Exclusion criteria included students who disclosed a diagnosis of anxiety or depression. The bachelor of medicine and bachelor of surgery program at the University of Ha'il, Saudi Arabia (SA), is a five-year program followed by a mandatory one-year internship. Each year, 80 male and female students are accepted into the program. An online survey (in English, the language of instruction in our school) was sent via SurveyMonkey (surveymonkey.com) to all 82 graduates. A brief explanation was provided, and anonymity and voluntary participation were emphasized. No incentives were offered for answering the survey. The survey was sent on April 22, 2020, and responses were collected over two weeks. At that time, graduates were finishing their last module (surgery) off-campus due to school closures.

The survey comprised three parts. Part one consisted of general demographic questions exploring age, gender, marital status, and city of origin, as well as questions on general health and COVID-19 diagnosis in self or others. Part two screened respondents for anxiety and depression utilizing the Generalized Anxiety Disorder 7-item (GAD-7) and the Public Health Questionnaire 9-item (PHQ-9) scales. Part three required respondents to rank five areas of concern during the COVID-19 pandemic from 1 (most important area of concern) to 5 (least important area of concern).

The GAD-7 is a validated 7-item self-report scale used to screen for generalized anxiety disorder [10]. Items are scored as " 0 ," " 1 ," “2," or “ 3 ," representing "not at all," "several days," "more than half the days," or "nearly every day," respectively. Possible scores range from 0 to 21 , with scores of 0 to 4 categorized as minimal anxiety, 5 to 9 mild anxiety, 10 to 14 moderate anxiety, and 15 to 21 severe anxiety. A meta-analysis has shown that the use of 8 as a cutoff score provided reasonable sensitivity of $0.83(0.71-0.91)$ at $95 \%$ confidence interval (CI) and a specificity of 0.84 (0.70 to $0.92)$ at the same CI [11].

The PHQ-9 is a validated 9-item scale, widely used to screen for depression in nonpsychiatric settings [12]. The scale was initially constructed as a self-report version of the Primary Care Evaluation of Mental Disorders PRIME-MD [13]. Items are scored as " 0, , " 1, ," " 2 ," or " 3 ," representing "not at all," "several days," "more than half the days," or "nearly every day," respectively. Possible scores range from 0 to 27 with scores of 0 to 4 categorized as minimal depression, 5 to 9 mild depression, 10 to 14 moderate depression, 15 to 19 moderately severe depression, and 20 to 27 severe depression [12]. A recent individual participant data metaanalysis showed that the use of 10 as a cutoff score maximized both the sensitivity of the scale to $0.88(0.83-0.92)$ at $95 \%$ CI and specificity to $0.85(0.82$ to 0.88$)$ at the same CI [14].

Data were analyzed using IBM SPSS Statistics (Version 26) predictive analytic software. Means and standard deviation were used to describe continuous data; frequency and percentages were used for categorical data. Respondents were then divided into three subgroups, based on the results of the anxiety and depression screen. A GAD-7 $\geq 8$ and a PHQ-9 $\geq 10$ were used as cutoff results for anxiety and depression, respectively. Group one screened positive for anxiety, and group two screened positive for depression. The third group screened positive for both anxiety and depression. The Shapiro-Wilk's test $(p>0.05)$ was used to test normal distribution of the data. Due to the expected sample size, the Fisher's exact test of independence was applied. The unpaired (independent) $t$-test was used to compare scores among the three groups. Statistical significance was set at $p<0.05$ (two-tailed).

\section{Results}

The survey was sent to 43 male and 39 female graduates (class of 2020). Fifty-five students completed the online survey giving a $67 \%$ response rate. Two respondents were excluded due to a disclosed diagnosis of depression. The mean age was $23.91(\mathrm{SD}=0.66)$. Most respondents lived with a family member (96\%); only a few had parents living in another region (15\%). In regard to general health, $6 \%$ of respondents had a chronic health condition, and $11 \%$ disclosed a diagnosis of COVID-19 in self, relative, or an acquaintance.

We aimed to determine the point prevalence of anxiety and depression among the cohort. Anxiety symptoms were present in $34 \%$ of students, depression was present in $26 \%$, and $19 \%$ had both depression and anxiety symptoms. Table 1 summarizes demographical data and general health data for each of the three subgroups. Symptoms of anxiety were significantly higher among female graduates (54 vs. $15 \%, p=0.004$ ).

Figure 1 shows the box and whisker plot diagrams for GAD-7 (a) and PHQ-9 (b) scores stratified by gender. The unpaired (independent) $t$-test was used to compare mean GAD-7 and PHQ-9 scores between male and female students. Female students $(M=8.85, \mathrm{SD}=5.446)$ had significantly higher GAD-7 scores compared to male students $(M=4.59$, $\mathrm{SD}=3.544), t(51)=-3.383, p=0.001$. No significant differences were observed between the PHQ-9 scores for female students $(M=7.15, \mathrm{SD}=4.514)$ compared to male students $(M=6.48, \mathrm{SD}=4.972), t(51)=-0.515, p=0.609$.

Depression was classified as absent to minimal in $38 \%$, mild in $36 \%$, moderate in $19 \%$, and moderately severe in $8 \%$. Anxiety was classified as absent to minimal in 34\%, mild in $49 \%$, moderate in $8 \%$, and severe in $9 \%$. Bivariate correlation with Pearson's $r$ showed that anxiety and depression scores were significantly correlated, $r(51)=0.65, p<0.01$. Figure 2 shows the scatter plot diagram for individual anxiety and depression scores with evident positive correlation.

The highest-ranked concerns were the possible effect of the pandemic on the forthcoming internship year and on the current academic course, as seen in Figure 3.

\section{Discussion}

The mental health repercussions of the COVID-19 pandemic have been documented from different aspects: from fear of the disease itself to the effects of containment 
TABLE 1: Demographic characteristics of participants overall and of the three subgroups.

\begin{tabular}{|c|c|c|c|c|c|c|c|}
\hline \multirow[t]{2}{*}{ Variables } & \multirow{2}{*}{$\begin{array}{c}\text { Total sample }(N=53) \\
\qquad N(\%)\end{array}$} & \multicolumn{2}{|c|}{$\begin{array}{c}\text { GAD-7 } \geq 8 \\
(N=18)\end{array}$} & \multicolumn{2}{|c|}{$\begin{array}{l}\text { PHQ-9 } \geq 10 \\
(N=14)\end{array}$} & \multicolumn{2}{|c|}{$\begin{array}{c}\text { GAD }-7 \geq 8 \text { and } \\
\text { PHQ- } 9 \geq 10 \\
(N=10)\end{array}$} \\
\hline & & $N(\%)$ & $p$ & $N(\%)$ & $p$ & $N(\%)$ & $p$ \\
\hline \multicolumn{8}{|l|}{ Gender } \\
\hline Female & $26(49)$ & $14(54)$ & \multirow{2}{*}{0.004} & $7(27)$ & \multirow{2}{*}{1.000} & $6(23)$ & \multirow{2}{*}{0.501} \\
\hline Male & $27(51)$ & $4(15)$ & & $7(26)$ & & $4(15)$ & \\
\hline \multicolumn{8}{|l|}{ Marital status } \\
\hline Single & $50(94)$ & $18(36)$ & 0.543 & $14(28)$ & 0.557 & $10(20)$ & 1.000 \\
\hline Parents live in the same region & $45(85)$ & $13(29)$ & 0.104 & $11(24)$ & 0.422 & $7(16)$ & 0.163 \\
\hline Lives with a family member & $51(96)$ & $17(33)$ & 1.000 & $13(26)$ & 0.462 & $9(18)$ & 0.345 \\
\hline Chronic health condition & $3(6)$ & $2(67)$ & 0.263 & $1(33)$ & 1.000 & $1(33)$ & 0.473 \\
\hline COVID-19 diagnosis in self & $2(4)$ & $1(50)$ & 1.000 & $1(50)$ & 0.462 & $1(50)$ & 0.345 \\
\hline COVID-19 diagnosis in a relative or acquaintance & $4(8)$ & $2(50)$ & 0.598 & $2(50)$ & 0.282 & $2(50)$ & 0.157 \\
\hline
\end{tabular}

$N$, number; GAD-7, Generalized Anxiety Disorder 7-item scale; PHQ-9, Patient Health Questionnaire 9-item scale; $p$, $p$ value using Fisher's exact test (2sided); COVID-19, corona virus disease 2019.

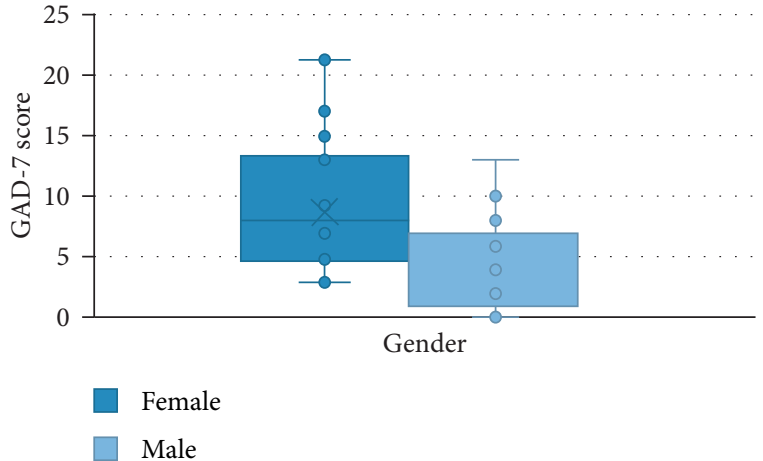

(a)

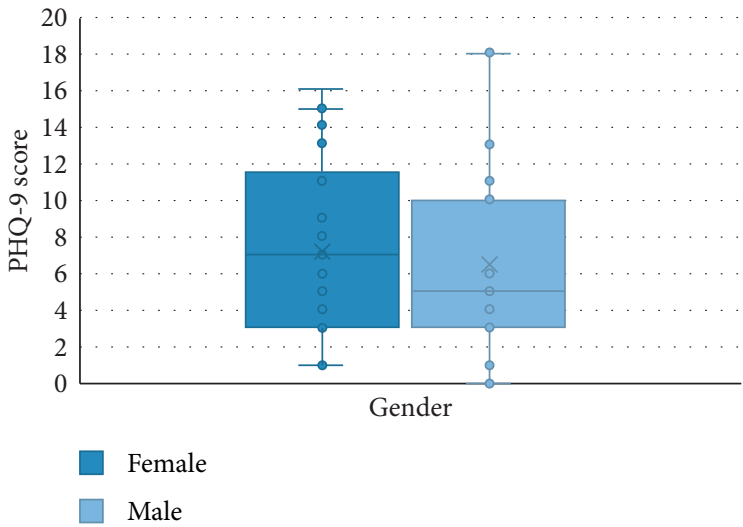

(b)

FIGURE 1: Box and whisker plot diagrams for GAD-7 (a) and PHQ-9 scores (b) stratified by gender. GAD-7, Generalized Anxiety Disorder 7item scale; PHQ-9, Patient Health Questionnaire 9-item scale.

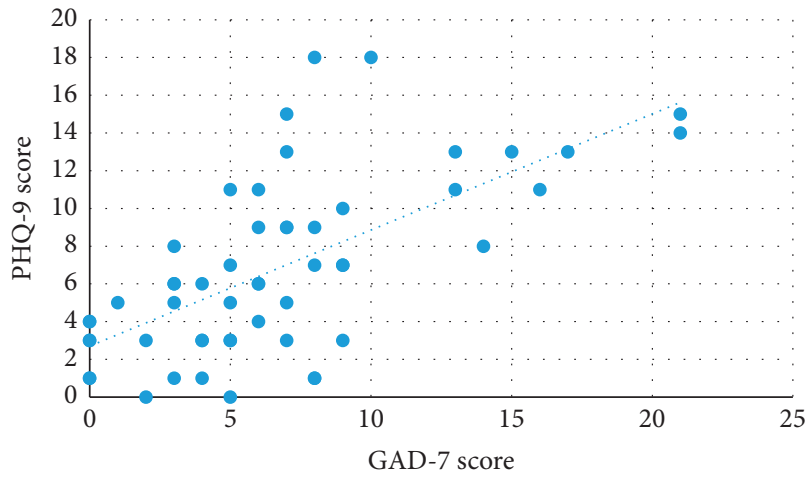

FIgURE 2: Scatter plot diagram displaying positive correlation between individual GAD-7 and PHQ-9 scores. GAD-7, Generalized Anxiety Disorder 7-item scale; PHQ-9, Patient Health Questionnaire 9-item scale.

measures such as quarantine and lockdown. An early study from China on the general public conducted two weeks within the outbreak of COVID-19 showed that $16.5 \%$ of

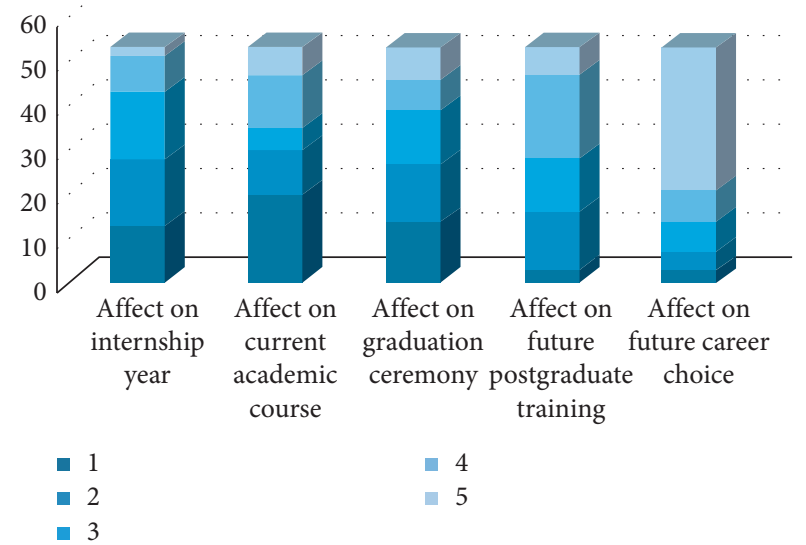

FIgURE 3: Areas of graduates' perceived concern ranked from 1 (highest concern) to 5 (least concern).

respondents reported symptoms of moderate to severe depression, and $28.8 \%$ reported symptoms of moderate to severe anxiety (utilizing the Depression, Anxiety, and Stress 
Scale (DASS-21)) [15]. In the present study, we found a point prevalence of $34 \%$ for anxiety and $26 \%$ for depression.

The impact of school closures on the psychological health of students was anticipated as much as its effect on their education [4]. In a longitudinal study over the past two years, researchers looked at the mental health of undergraduate college students and found an increase in anxiety and depression symptoms during the Winter 2020 term compared to previous academic terms [16]. Symptoms of depression were reported in $19 \%$ of college students from six universities in China using PHQ-9 score $\geq 10$. Students in their final years were found to have significantly higher depression scores [17].

The first confirmed case of COVID-19 in Saudi Arabia was announced on March 2nd, and nationwide school closures took effect on March 9th. Our study was conducted six weeks after the initiation of school closures; students were less than two months away from graduating from medical school. Symptoms of anxiety were present in $34 \%$ (GAD-7 score $\geq 8$ ) of surveyed students, while symptoms of depression (PHQ-9 score $\geq 10$ ) were present in $26 \%$. Cutoff scores were chosen according to recent meta-analyses $[11,14]$ that showed that their use increased the sensitivity and specificity of the scales. Original scoring of the scales and different cutoff points were used where appropriate to facilitate comparisons to other studies.

In a study on medical students during the pandemic, $24.9 \%$ had some symptoms of anxiety (GAD-7 score $\geq 1$ ) [18]. This differs vastly with our results, in which $96 \%$ of students had some symptoms of anxiety using the same criteria. Another study on medical students showed that symptoms of anxiety (GAD-7 score $\geq 5$ ) were present in $22.1 \%$ of students during the pandemic [19]. In our study, $66 \%$ have symptoms of anxiety at the same cutoff point. In the same study, 35.5\% had symptoms of depression (PHQ-9 score $\geq 5$ ), compared to $62 \%$ in our study.

The high proportion of anxiety and depression symptoms in our study ( $66 \%$ vs. $22.1 \%$ for anxiety and $62 \%$ vs. $35.5 \%$ for depression) has many possible explanations. Our study population comprised only medical students in their final year of study who are reaching an important milestone in their journey. After graduation, students start their internship training and prepare for further postgraduate education or seek job opportunities. Another possible reason is timing, as our study was conducted later in the pandemic, during which more cases and case fatalities were reported, also students were two weeks away from their surgery final exams and graduation.

Inherent differences between the study populations are a crucial point to consider as well. There are no current studies observing anxiety or depression symptoms among Saudi medical students during the pandemic. A study from 2018 that targeted medical students from almost all medical schools in Saudi Arabia (including 109 students from our institution) has shown that $63 \%$ had depression symptoms (PHQ-9 score $\geq 5$ ) [20], almost replicated by our finding of $62 \%$. Other single institute studies have shown a variable prevalence for anxiety, from $15.5 \%$ [21] to $64 \%$ [22] using different screening scales.
Our study findings report higher rates of anxiety and depression symptoms among graduating medical students during the COVID-19 pandemic compared to medical students in China $[18,19]$. On the other hand, our results support previous findings on the baseline prevalence of depression and anxiety among medical students in Saudi Arabia [23]. A closer look is warranted at the reasons for the higher prevalence of mental health problems compared to medical students in other countries, as well as implementing structured programs to help students overcome these mental health concerns.

\section{Conclusions}

Our study supports the high prevalence of depression and anxiety symptoms among Saudi medical students. The aim was to explore the effects of the COVID-19 epidemic on medical students' mental health in their final year of study. Although graduates scored higher in validated scales used to screen for depression and anxiety compared to other studies utilizing the same scales in medical students, scores were similar to studies conducted before the pandemic. Our study is limited by the small study population, and large-scale studies are warranted to explore baseline mental health of medical students as well as a closer look at the effects of crises on baseline mental health. Meanwhile, psychological support should be offered to students to help overcome any concerns they might endure during this epidemic. As the pandemic continues to impact education, more efforts should be put into mitigating the detrimental effect it has on students.

\section{Data Availability}

Data used to support the findings of this study are available from the author upon request.

\section{Conflicts of Interest}

The author declares that there are no conflicts of interest regarding the publication of this paper.

\section{References}

[1] N. Zhu, D. Zhang, W. Wang et al., "A novel Coronavirus from patients with pneumonia in China, 2019," New England Journal of Medicine, vol. 382, no. 8, pp. 727-733, 2020.

[2] WHO, WHO Director-General's Opening Remarks at the Media Briefing on COVID-19, WHO, Geneva, Switzerland, 2020, https://www.who.int/dg/speeches/detail/who-directorgeneral-s-opening-remarks-at-the-media-briefing-on-covid19---11-march-2020.

[3] UNESCO, COVID-10 Impact on Education, UNESCO, Paris, France, 2020, https://en.unesco.org/covid19/ educationresponse.

[4] P. Sahu, "Closure of universities due to Coronavirus disease 2019 (COVID-19): impact on education and mental health of students and academic staff," Cureus, vol. 12, no. 4, p. e7541, 2020. 
[5] D. G. Miller, L. Pierson, and S. Doernberg, "The role of medical students during the COVID-19 pandemic," Annals of Internal Medicine, vol. 173, no. 2, pp. 145-146, 2020.

[6] D. Soled, S. Goel, D. Barry et al., "Medical student mobilization during a crisis: lessons from A COVID-19 medical student response team," Academic Medicine, vol. 95, no. 9, pp. 1384-1387, 2020.

[7] L. Jeong, Z. Smith, A. Longino, S. E. Merel, and K. McDonough, "Virtual peer teaching during the COVID-19 pandemic," Medical Science Educator, vol. 3, no. 4, pp. 1361-1362, 2020.

[8] T. T. Quek, W. W. Tam, B. X. Tran et al., "The global prevalence of anxiety among medical students: a metaanalysis," International Journal of Environmental Research and Public Health, vol. 16, no. 15, p. 2735, 2019.

[9] L. S. Rotenstein, M. A. Ramos, M. Torre et al., "Prevalence of depression, depressive symptoms, and suicidal ideation among medical students," JAMA, vol. 316, no. 21, pp. 2214-2236, 2016.

[10] R. L. Spitzer, K. Kroenke, J. B. W. Williams, and B. Löwe, "A brief measure for assessing generalized anxiety disorder," Archives of Internal Medicine, vol. 166, no. 10, pp. 1092-1097, 2006.

[11] F. Plummer, L. Manea, D. Trepel, and D. McMillan, "Screening for anxiety disorders with the GAD-7 and GAD-2: a systematic review and diagnostic metaanalysis," General Hospital Psychiatry, vol. 39, pp. 24-31, 2016.

[12] K. Kroenke, R. L. Spitzer, and J. B. W. Williams, "The PHQ-9," Journal of General Internal Medicine, vol. 16, no. 9, pp. 606-613, 2001.

[13] R. L. Spitzer, K. Kroenke, and J. B. Williams, "Validation and utility of a self-report version of PRIME-MD," JAMA, vol. 282, no. 18, pp. 1737-1744, 1999.

[14] B. Levis, A. Benedetti, and B. D. Thombs, "Accuracy of Patient Health Questionnaire-9 (PHQ-9) for screening to detect major depression: individual participant data meta-analysis," $B M J$, vol. 365, p. I1476, 2019.

[15] C. Wang, R. Pan, X. Wan et al., "Immediate psychological responses and associated factors during the initial stage of the 2019 Coronavirus disease (COVID-19) epidemic among the general population in China," International Journal of Environmental Research and Public Health, vol. 17, no. 5, 2020.

[16] J. F. Huckins, A. W. DaSilva, W. Wang et al., "Mental health and behavior during the early phases of the COVID-19 pandemic: a longitudinal mobile smartphone and ecological momentary assessment study in college students," Journal of Medical Internet Research, vol. 22, no. 6, Article ID e20185, 2020.

[17] W. Tang, T. Hu, B. Hu et al., "Prevalence and correlates of PTSD and depressive symptoms one month after the outbreak of the COVID-19 epidemic in a sample of home-quarantined Chinese University students," Journal of Affective Disorders, vol. 274, pp. 1-7, 2020.

[18] W. Cao, Z. Fang, G. Hou et al., "The psychological impact of the COVID-19 epidemic on college students in China," Psychiatry Research, vol. 287, Article ID 112934, 2020.

[19] J. Liu, Q. Zhu, W. Fan, J. Makamure, C. Zheng, and J. Wang, "Online mental health survey in a medical college in China during the COVID-19 outbreak," Frontiers in Psychiatry, vol. 11, 2020.

[20] H. Alharbi, A. Almalki, F. Alabdan, and B. Haddad, "Depression among medical students in Saudi medical colleges: a cross-sectional study," Advances in Medical Education and Practice, vol. 9, pp. 887-891, 2018.
[21] A. H. El-Gilany, M. Amr, and S. Hammad, "Perceived stress among male medical students in Egypt and Saudi Arabia: effect of sociodemographic factors," Annals of Saudi Medicine, vol. 28 , no. 6 , pp. 442-448, 2008.

[22] B. Kulsoom and N. A. Afsar, "Stress, anxiety, and depression among medical students in a multiethnic setting," Neuropsychiatric Disease and Treatment, vol. 11, pp. 1713-1722, 2015.

[23] M. Z. Ahmed, O. Ahmed, Z. Aibao, S. Hanbin, L. Siyu, and A. Ahmad, "Epidemic of COVID-19 in China and associated psychological problems," Asian Journal of Psychiatry, vol. 51, Article ID 102092, 2020. 